\title{
Jump Forward to Get Back to Basics
}

\author{
Eric B. Bass, MD, MPH \\ Division of General Internal Medicine, Johns Hopkins University, Baltimore, MD, USA.
}

J Gen Intern Med 30(11):1577-8

DOI: $10.1007 / \mathrm{s} 11606-015-3444-\mathrm{x}$

(c) Society of General Internal Medicine 2015

$\mathrm{H}$ ow should primary care providers react to the study by Sutkowi-Hemstreet et al. showing that many patients do not understand the benefits and harms of "overused" screening tests? ${ }^{1}$ My instinctual reaction as a general internist is to aspire to help patients make appropriate decisions about screening tests by being a trustworthy source of information and an effective counselor. I must confess, however, that I never have enough time in my practice to fully explain what is known about all potential harms and benefits of each screening test that could be offered to a patient.

In a classic study published in 1999, Braddock et al. found that only $9 \%$ of decisions made in routine office visits with primary care physicians and surgeons met a reasonable set of criteria for informed decision making. ${ }^{2}$ These seven criteria included 1) discussion of the patient's role in decision making; 2) discussion of the clinical issue or nature of the decision; 3 ) discussion of the alternatives; 4) discussion of potential benefits and risks of the alternatives; 5) discussion of uncertainties associated with the decision; 6) assessment of the patient's understanding; and 7) exploration of patient preference. Sixteen years after that study was published, my review of the criteria provides a sobering self-assessment of the adequacy of my counseling about screening tests. How often do your discussions with patients about screening tests meet all of the criteria for informed decision making? Does the adequacy of your approach to informed decision making differ based on whether a screening test is recommended or not recommended?

Much attention is now focused on overuse of health services, thanks in large part to the Choosing Wisely campaign launched by the American Board of Internal Medicine (ABIM) Foundation. ${ }^{3}$ One of the most remarkable aspects of the Choosing Wisely campaign is its success in engaging physicians from more than 50 different specialty societies, indicating broad support for involving patients in discussions about potentially unnecessary tests and procedures. Many of the Choosing Wisely recommendations focus on screening tests such as those considered in the study by SutkowiHemstreet et al. For example, the American College of

Published online June 30, 2015
Physicians recommends against obtaining a screening exercise electrocardiogram in individuals who are asymptomatic and at low risk for coronary heart disease. ${ }^{3}$ Of course, the success of these types of recommendations ultimately will depend on the ability to promote conversations between patients and physicians about opportunities to reduce unnecessary care.

Although the principles of informed decision making apply as much to efforts to reduce unnecessary screening as they do to increasing the use of recommended screening, human psychology makes it difficult for people to forego widely used practices, even when current evidence shows they have little value. As Roman and Asch suggested in their article about the challenge of de-adopting low-value care, conversations about low-value services should account for psychological forces such as optimism bias (being more willing to accept new information when it is in one's favor), confirmation bias (favoring information that confirms prior beliefs), loss aversion (feeling more strongly about losses than gains), and the affect heuristic (when initial feelings color judgment of risks and benefits). ${ }^{4}$ In light of how people view losses relative to gains, it may be particularly important to explain all potential harms of screening tests that have low value. Such conversations are consistent with the ethics of parsimonious medicine, which seeks to deliver appropriate care tailored to the needs of patients, while avoiding wasteful or harmful services. ${ }^{5}$

The problem for primary care providers is that it would be overwhelming to conduct a high-quality one-on-one conversation with every patient about every preventive service that has been recommended or not recommended. Using the Electronic Preventive Services Selector tool developed by the Agency for Healthcare Research and Quality, I found that a 57-year-old male non-smoker would need to consider 14 preventive services recommended by the U.S. Preventive Services Task Force, as well as 13 preventive services that are not recommended, and 27 preventive services associated with uncertain recommendations. ${ }^{6}$ As much as I share Dr. Braddock's desire to get back to the basics of informed decision making, it will be impossible to meet the needs of our patients for all of these conversations without much better system-level support.

It is time to jump forward with new ways of facilitating conversations about high-value and low-value screening tests. The Choosing Wisely campaign has done a great job in raising public awareness about the challenge of reducing unnecessary care. Our clinical practices should capitalize on the public demand for information by making greater use of consumer- 
friendly learning aids and decision support tools that can help patients distinguish between unnecessary care and recommended screening services. Even with well-designed learning aids, however, many patients will still need to communicate with a trusted health professional about how the recommendations apply to their individual circumstances.

Systems should be designed to educate patients about relevant screening tests and to facilitate communication between patients and their primary care providers about the tests in a manner that accounts for individual characteristics and preferences. The tools exist to support better communication if we abandon unrealistic expectations of primary care providers, who will not have time to incorporate discussion of all screening services in face-to-face visits. Krist et al. have provided a wonderful example of the forward-thinking work that is needed by developing an interactive preventive health record that promotes shared decision making about preventive services. ${ }^{7}$ In addition to demonstrating the feasibility of the information technology tool, the authors have launched a trial to assess the effectiveness of implementing the technology in a large number of practices, with an emphasis on measuring engagement of disadvantaged patients. Another example of a jump forward is the BETTER trial-Building on Existing Tools to Improve Chronic Disease Prevention and Screening in Primary Care. ${ }^{8}$ The pragmatic BETTER trial demonstrated that it is possible to improve delivery of preventive services by using a "Prevention Practitioner" to facilitate hour-long conversations with patients about appropriately tailored recommendations for preventive care. Fortunately, new payment models are emerging that will help to support these kinds of services for promoting high-value care. Since many patients will look to their primary physician for advice on the bewildering array of recommendations about screening tests, the systems should be designed to preserve a role for the primary care provider in responding to questions that patients will have when they receive appropriate information about the options. Moreover, training programs should aspire to give the next generation of physicians a high level of competency in an inter-disciplinary approach to communicating with patients about screening tests. One of the most promising initiatives in this regard is the recently announced commitment to high-value care education by the Alliance for Academic Internal Medicine, ABIM, ABIM Foundation, and American College of Physicians. ${ }^{9}$ As clinicians, educators, and investigators, we should support their commitment to jump forward with new models for facilitating communication about high-value care and its thorny counterpart, low-value care.

Corresponding Author: Eric B. Bass, MD, MPH; Division of General Internal MedicineJohns Hopkins University, Baltimore, MD, USA (e-mail: ebass@jhmi.edu).

\section{REFERENCES}

1. Sutkowi-Hemstreet A, Vu M, Harris R, Brewer NT, Dolor RJ, Sheridan SL. Adult patients' perspectives on the benefits and harms of overused screening tests: a qualitative study. J Gen Intern Med. 2015. doi:10.1007/ s11606-015-3283-9.

2. Braddock CH, Edwards KA, Hasenberg NM, Laidley TL, Levinson W. Informed decision making in outpatient practice. Time to get back to basics. JAMA. 1999;282:2313-20.

3. Wolfson D, Santa J, Slass L. Engaging physicians and consumers in conversations about treatment overuse and waste: a short history of the choosing wisely campaign. Acad Med. 2014;89:990-5.

4. Roman BR, Asch DA. Faded promises: the challenge of deadopting lowvalue care. Ann Intern Med. 2014;161:149-50.

5. Tilburt JC, Cassel CK. Why the ethics of parsimonious medicine is not the ethics of rationing. JAMA. 2013;309:773-4.

6. ePSS (Electronic Preventive Services Selector). Available at: http://epss. ahrq.gov/PDA/about.jsp [accessed on May 25, 2015].

7. Krist AH, Aycock RA, Etz RS, Devoe JE, Sabo RT, Williams R, Stein KL, Iwamoto G, Puro J, Deshazo J, Kashiri PL, Arkind J, Romney C, Kano M, Nelson C, Longo DR, Wolver S, Woolf SH. MyPreventiveCare: implementation and dissemination of an interactive preventive health record in three practice-based research networks serving disadvantaged patients-a randomized cluster trial. Implement Sci. 2014;9:181.

8. Grunfeld E, Manca D, Moineddin R, Thorpe KE, Hoch JS, CampbellScherer D, Meaney C, Rogers J, Beca J, Krueger P, Mamdani M, for the BETTER Trial Investigators. Improving chronic disease prevention and screening in primary care: results of the BETTER pragmatic cluster randomized controlled trial. BMC Fam Pract. 2013;14:175.

9. Smith CD, Levinson WS, on behalf of the Internal Medicine HVC Advisory Board. A commitment to high-value care education from the internal medicine community. Ann Intern Med. 2015;162:639-40. 